\title{
Pseudolipomatose do Cólon: Relato de Caso
}

\author{
Pseudolipomatosis of the Colon: A Case Report
}

\section{CARLOS AUGUSTO REAL MARTINEZ; CARLOS ALBERTO FONTES DE SOUZA ${ }^{2}$; MARCELO RAMOS NORONHA ${ }^{3}$; CAMILAHELAEHILALFREDO ${ }^{4}$; ANAPAULAPIMENTEL SPADARI ${ }^{4}$; PRISCILLACECÍLIAMENDES BARTOCCI ${ }^{4}$; DENISEGONÇALVES PRIOLLI ${ }^{1}$}

\author{
${ }^{1}$ Professor Adjunto Doutor do Programa de Pós Graduação em Ciências da Saúde da Universidade São Francisco, \\ Bragança Paulista (SP); ${ }^{2}$ Professor Assistente da Disciplina de Anatomia Patológica do Curso de Medicina da \\ Universidade São Francisco, Bragança Paulista (SP); ${ }^{3}$ Médico Residente do $2^{\circ}$ ano do Serviço de Anatomia Patológica \\ do Hospital Universitário São Francisco, Bragança Paulista (SP); ${ }^{4}$ Acadêmicas do Curso de Medicina da Universidade \\ São Francisco, Bragança Paulista (SP).
}

MARTINEZ CAR; SOUZA CAF; NORONHA MR; ALFREDO CH; SPADARI APP; BARTOCCI PCM; PRIOLLI DG. Pseudolipomatose do Cólon: Relato de Caso. Rev bras Coloproct, 2008;28(1): 104-107.

RESUMO: A pseudolipomatose cólica é uma condição benigna, raramente descrita, caracterizada histologicamente pela presença de múltiplas vesículas contendo ar na parede do intestino grosso. Acredita-se que a doença tenha origem iatrogênica, ocasionada pela infiltração de ar através da mucosa cólica durante a realização de procedimentos endoscópicos. O objetivo do presente relato é apresentar um caso de pseudolipomatose cólica, confirmada por meio de estudo histopatológico e imunoistoquímico. Relato do caso: Mulher, 74 anos foi submetida à colonoscopia para investigação de anemia. Apresentava sangramento retal cíclico durante as evacuações, perda ponderal e anemia. $O$ exame endoscópico não mostrou alterações do cólon, detectando apenas mamilo hemorroidário que no momento do exame apresentava sinais de sangramento recente. Três semanas após o exame foi submetida à ligadura elástica do mamilo hemorroidário. Durante a realização do procedimento encontrou-se área sobrelevada na mucosa intestinal, de coloração amarelo-esbranquiçada, com aproximadamente quatro centímetros de diâmetro, localizada na parede posterior do reto, a $13 \mathrm{~cm}$ da margem anal que inexistia quando da realização da colonoscopia. Foi realizada biópsia da região alterada da mucosa e ligadura elástica do mamilo hemorroidário. $O$ exame histopatológico demonstrou a presença de inúmeros vacúolos de diâmetro variado, algumas vezes confluentes, localizados na lamina própria da parede intestinal mimetizando tecido adiposo. Notava-se ainda presença de pertuito longitudinal que comunicava a região vacuolizada com a superfície da mucosa cólica. As técnicas histoquímicas e imunoistoquímicas para detecção de gordura no interior das vesículas revelaram-se negativas, permitindo o diagnóstico de pseudolipomatose cólica. Após a ligadura elástica a enferma não mais apresentou sangramento retal, recuperando-se da anemia. Conclusão: A pseudolipomatose do cólon, apesar da raridade, é uma condição que pode ser encontrada após a realização de procedimentos endoscópicos no cólon.

Descritores: Colo; Colonoscopia/efeitos adversos; Lipomatose; Endoscopia; Pseudolipomatose.

\section{INTRODUÇÃO}

A pseudolipomatose cólica (PLC) é uma condição benigna, raramente descrita, de curso geralmente assintomático, caracterizada do ponto de vista histológico pela presença de pequenas vesículas com conteúdo gasoso localizadas na lâmina própria da pa- rede do intestino grosso de enfermos previamente submetidos a exames endoscópicos. ${ }^{1-6}$ A nomenclatura da doença foi proposta em 1985 pelo aspecto similar com a infiltração gordurosa da parede cólica observada na lipomatose da mucosa intestinal. ${ }^{1}$ Apresenta etiologia incerta acreditando-se que possa ocorrer em consequiência à infiltração de ar na parede intestinal, a partir de solu-

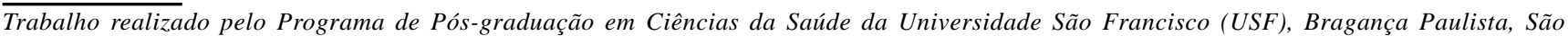
Paulo - Brasil. 
ções de continuidade na mucosa cólica ocasionadas por traumatismos durante a realização do procedimento endoscópico. ${ }^{1,2,6,8} \mathrm{~A}$ enfermidade também ocorre no estômago e duodeno apresentando prevalência estimada de 0,02 a 1,7\% dos exames endoscópicos. ${ }^{5}$ Estima-se que existam pouco mais de 60 casos publicados de pseudolipomatose acometendo o cólon. ${ }^{6}$

O objetivo do presente artigo é apresentar um caso de PLC após realização de exame endoscópico retal, confirmado por estudo histoquímico e imunoistoquímico.

\section{RELATO DO CASO}

A publicação deste relato foi autorizada pelo Comitê de Ética da Universidade São Francisco (CAAE: 0010.0142.000-07) após Termo de Consentimento assinado pela doente.

Mulher, 74 anos, branca, obesa, com antecedentes de hipertensão arterial e hipertireodismo, procurou atendimento queixando-se de fraqueza, inapetência, sangramento retal cíclico as evacuação e perda ponderal há duas semanas. Ao exame físico encontrava-se em REG, descorada $++/+++$, pulso de 90 b.p.m., PA 160 x $100 \mathrm{mmHg}$. O toque retal revelava presença de sangue vivo na luva. Apresentava ao eritrograma hemoglobina de $9,6 \mathrm{~g} / \mathrm{dl}$ além de microcitose e hipocrômia. $\mathrm{O}$ exame proctológico identificava mamilos hemorroidários de II grau. A colonoscopia confirmava o diagnóstico de doença hemorroidária, sendo o restante do cólon normal. Foi submetida à ligadura elástica para tratamento da doença. Durante a retoscopia para a realização do procedimento, encontrou-se na superfície mucosa da parede posterior do reto a aproximadamente $13 \mathrm{~cm}$ da margem anal, área irregular, sobrelevada, de coloração amareloesbranquiçada sem sinais de sangramento, não identificada na colonoscopia prévia. Realizou-se biópsia do local. O estudo histopatológico do fragmento biopsiado demonstrou presença de pertuito comunicando a luz intestinal com a camada submucosa. Neste local, notava-se a formação de múltiplos vacúolos, que variavam de tamanho entre 50 a 400 micra de diâmetro, mimetizando tecido adiposo e sugerindo, inicialmente, o diagnóstico de lipomatose da parede do reto (Figuras 1 e 2). Notava-se ainda no córion, infiltrado inflamatório linfoplasmocitário, edema e discreta fibrose. A técnica histoquímica do Sudan negro específica para gordura não mostrava presença de lipídios no interior dos vacúolos. O estudo imunoistoquímico realizado pela técnica da avidina-biotina-peroxidase (ABC), utilizando anticorpo primário anti-proteína S-100, (Dako, Copenhagen, polyclonal rabbit Anti-S100), confirmava a inexistência de tecido adiposo no interior das vesículas, permitindo o diagnóstico de PLC.

Após a realização da ligadura elástica a doente não apresentou mais episódios de sangramento retal, recuperando seu peso inicial e a anemia. A retoscopia de controle realizada seis meses após o diagnóstico de PLC, não demonstrou mais a presença do mamilo hemorroidário, bem como a alteração da mucosa cólica anteriormente descrita. No momento a enferma encontra-se assintomática, 14 meses após o diagnóstico.



Figura 1 - Formação de vesículas nas camadas mucosa e submucosa da parede cólica - seta (HE-100x).



Figura 2 - Formação de vesículas nas camadas mucosa e submucosa da parede cólica - seta (HE-400x). Vacuolização da submucosa do cólon (HE 400x). 


\section{DISCUSSÃO}

A PLC foi descrita pela primeira vez por Snover et al., em 1985, que caracterizaram a enfermidade como a presença de vesículas com conteúdo gasoso na lâmina própria da parede do intestino grosso. ${ }^{1} \mathrm{~A}$ doença foi assim denominada pelo aspecto histológico semelhante ao da lipomatose intestinal, em virtude dos vacúolos gasosos encontrados na mucosa retal mimetizarem ao exame microscópico os adipócitos presentes na submucosa de portadores de lipomatose cólica. ${ }^{1}$

A PLC é uma enfermidade rara, estimando-se que existam aproximadamente 60 casos publicados. ${ }^{1,2,3,9-13}$ Estudos revisando grandes séries revelam que a PLC pode ocorrer em cerca de $0,02 \%$ a $1,7 \%$ dos pacientes submetidos a exames endoscópicos, incidindo com maior frequiência entre a sexta e sétima década de vida, provavelmente por se tratar do grupo etário onde os exames endoscópicos do cólon são realizados com maior freqüência. ${ }^{1-6}$ É provável que a incidência da doença encontre-se subestimada pelo curso assintomático apresentado pela maioria dos doentes. ${ }^{10}$ Em relação ao sexo a prevalência é assunto controverso, pois enquanto alguns autores encontram maior acometimento do gênero feminino, outros descrevem maior prevalência no masculino. ${ }^{1-3,6}$

A etiopatogenia da PLC permanece obscura, parecendo tratar-se muito mais de complicação do exame endoscópico do que uma enfermidade propriamente dita. ${ }^{6}$ Atualmente, a teoria etiopatogênica mais aceita é que a doença tenha origem iatrogênica, resultante do barotrauma ocasionado pela penetração de gás na mucosa intestinal durante a realização de exames endoscópicos. ${ }^{2,410}$ A possibilidade de tratar-se de doença iatrogênica fica reforçada por estudos demonstrando que sua incidência é endoscopista dependente. ${ }^{1,12}$

Autores sugerem a relação entre PLC e utilização de endoscópios desinfetados com peróxido de hidrogênio, uma vez que foi possível reproduzir a PLC experimentalmente em ratos, por meio da infusão de peróxido de hidrogênio na mucosa cólica. ${ }^{14}$ Contudo, a possibilidade do aparecimento da doença em doentes submetidos à colonoscopia com equipamentos desinfetados com outros agentes desinfetantes, não confirmam esta proposta etiopatogênica. . $^{2-4,10-13}$

O aspecto endoscópico da PLC é bem característico. Macroscopicamente observam-se placas de mucosa de coloração amarelo-esbranquiçada, elevadas, levemente aderentes, únicas ou múltiplas, segmentadas ou localizadas. As placas possuem diâmetro variando de $0,2 \mathrm{~cm}$ a $5 \mathrm{~cm}$, situando-se com maior freqüência no cólon sigmóide e reto, padrões semelhantes aos encontrados na doente do presente relato. ${ }^{2,3,5}$

O exame microscópico apresenta invariavelmente os mesmos aspectos histológicos. Vacúolos opticamente vazios, algumas vezes coalescentes, geralmente localizados no córion entre as criptas da mucosa cólica. Na maior parte dos casos descritos os vacúolos medem entre 50 a $600 \mathrm{~mm}$ em seus maiores diâmetros, sendo habitualmente rodeados por infiltrado inflamatório mononuclear. ${ }^{1,2,6}$ Ocasionalmente é possível identificar soluções de continuidade na superfície epitelial da mucosa cólica próximo à lesão. Na doente do presente relato, a coloração pelo Sudan negro permitiu o diagnóstico diferencial com a lipomatose do cólon, pela inexistência de conteúdo gorduroso no interior das vesículas. Da mesma forma o estudo imunoistoquímico, com anticorpos primários antiproteína S-100, anti-CD31 e anti-CD34, pode confirmar que as vesículas não se encontravam preenchidas por tecido gorduroso, bem como não representavam dilatações vasculares. ${ }^{6}$ Estudo realizado anteriormente com emprego de microscopia eletrônica demonstrou que os vacúolos não são constituídos por fibras colágenas, bem como não encontramse limitados por uma membrana verdadeira, possuindo em seu interior conteúdo semelhante a linfa. ${ }^{6}$ De modo distinto a lipomatose os vacúolos da PLC, na maioria dos casos, geralmente desaparecem em cerca de três semanas. ${ }^{2}$

O diagnóstico diferencial da PLC deve ser feito com a pneumatose cistóide intestinal, onde a mucosa cólica apresenta-se com aspecto mamelonado e edemaciada, sugerindo ruptura das vesículas com retração da mucosa suprajacente. Ao exame histológico, a pneumatose cistóide apresenta vacúolos vazios restritos à submucosa, cercados por células gigantes multinucleadas e macrófagos, não atingindo a mucosa cólica a semelhança do que ocorre na PLC. ${ }^{15} \mathrm{O}$ linfangioma colônico embora possa assemelhar-se aos vacúolos da PLC apresentam coloração positiva em técnicas imunoistoquímicas que utilizam CD31 e CD $34 .{ }^{16}$

O tratamento da PLC é conservador, por se tratar de lesão benigna que desaparece na maioria dos casos em duas a três semanas sem deixar seqüelas. ${ }^{2}$ 
ABSTRACT: Pseudolipomatosis of the colon is a rarely described benign condition that is characterized histologically by the presence of multiple vesicles containing air, in the wall of the large intestine. It is believed that this disease has iatrogenic origin, caused by air infiltration through the colon mucosa during endoscopic procedures. The objective of the present report was to present a case of pseudolipomatosis of the colon that was confirmed by histopathological studies. Case report: A 74-year-old woman underwent colonoscopy to investigate anemia. She had been complaining about cyclical rectal bleeding during evacuations, weight loss and anemia. The endoscopic examination did not show any abnormalities in the colon and only showed the presence of a single hemorrhoid, which at the time of examination presented signs of recent bleeding. Three weeks after this examination, she underwent elastic ligation of the hemorrhoid. While carrying out this procedure, a whitish yellow raised area was found in the intestinal mucosa. It was approximately $4 \mathrm{~cm}$ in diameter and was located on the posterior wall of the rectum at a distance of $13 \mathrm{~cm}$ from the anal margin. It had not existed at the time of performing the colonoscopy. The altered region of the mucosa and the elastic ligation of the hemorrhoid were biopsied. Histopathological examination showed the presence of large numbers of vacuoles of varying diameters and sometimes confluent, located in the intestinal wall layer itself. The appearance mimicked adipose tissue. The presence of a longitudinal connection allowing communication between the vacuolized region and the surface of the colon mucosa was noted. Histochemical and immunohistochemical techniques for detecting fat inside the vesicles gave negative results, thus allowing the diagnosis of pseudolipomatosis of the colon. After the elastic rubber band ligation, the patient did not present any further rectal bleeding and recovered from the anemia. Conclusion: Although pseudolipomatosis of the colon is rare, it is a condition that may be found after performing endoscopic procedures in the colon.

Key words: Colon; Colonoscopy/adverse effects; Lipomatosis; Endoscopy; Pseudolipomatosis.

\section{REFERÊNCIAS}

1- Snover DC, Sandstad J, Hutton S. Mucosal Pseudolipomatosis of the colon. Am J. Clin. Pathol 1985; 84: 575-580.

2- Bem Rejeb A, Khedhiri F. Mucosal pseudo-lipomatosis of the colon. A propos of a case with a review of the literature. Arch Anat Cytol Pathol 1989;37:254-257.

3- Kaassis M, Croue A, Carpentier S, Burtin P, Boyer J. A case of colonic pseudolipomatosis: a rare complication of colonoscopy? Endoscopy 1997;29:325-7.

4- Witte J.T. Colonic Mucosal Pseudolipomatosis. Gastrointestinal Endoscopy 2001;54:750.

5- Nakasono M, Hirokawa M, Muguruma N, Okamura S, Iga A, Wada S, Okasaki M, Horie T, Fukuda T, Sano T. Colonic pseudolipomatosis, microscopically classified into two groups. Journal of Gastroenterology and Hepatology 2006,21:65-70.

6- Brevet M, Chatelain D, Bartoli E, Geslin G, Delcenserie R, Braillon A, Sevestre H, Dupas J.L. Colonic pseudolipomatosis: clinical, endoscopical and pathological features in nine cases.Gastroenterol Clin Biol 2006;30:9:9-13.

7- Pallarés Manrique H, Lara C, Maraver A, Talavera A, Ramos JL. Pseudolipomatosis duodenal . Rev Esp Enferm Dig 2002;94:42-3

8- Alper M, Akcan Y, Belini OK, Çukur S, Aksoy KA, Suna M. Gastric pseudolipomatosis, usual or unusual? Re-evaluation of 909 endoscopic gastric biopsies. Word J. Gastroenterol 2003;9:2846-48.

9- Prignet JM, Carloz E, Kunkel D. Pseudolipomatose muqueuse recto-sigmoidienne révelée par um diarrhée chronique. Discussion physiopathologique à propos d'un cas et reveu de la littérature. Acta endoscópica 1995;25:175-8
10- Warning JP, Manne RK, Wadas DD, Sanowski RA. Mucosal pseudolipomatosis: an air pressure-related colonoscopy complication. Gastrointest Endosc 1989;35:93-4.

11- Jonas G, Mahoney A, Murray J, Gertler S. Chemical colitis due to endoscope cleaning solutions: a mimic of pseudomembranous colitis. Gastroenterology 1988;95:1403-8.

12- Cox ME, Bostwick DG. Mucosal plaques seen at colonoscopy: chemical colitis or mucosal pseudolipomatosis? Gastroenterology 1989;96:1626-7.

13- Warning JP, Manne RK, Wadas DD. Mucosal plaques seen at colonoscopy: chemical colitis or mucosal pseudolipomatosis? Gastroenterology 1989;96:1626.

14- Sheehan JF, Brynjolfsson G. Ulcerative colitis following hydrogen peroxide enema: case report and experimental production with transient emphysema of colonic wall and gas embolism. Lab Invest 1960;9:150-68.

15- Gagliardi G, Thompson IW, Hershman MJ, Forbes A, Hawley PR, Talbot IC. Pneumatosis coli: a prposed pathogenesis based on study of 25 cases and review of the literature. Int J Colorectal Dis 1996;11:111-8.

16- Sato K, Maekawa T, Yabuki K, Tomita N, Eguchi M, Matsumoto M. Cystic lymphangiomas of the colon. J Gastroenterol 1999;34:520-4.

Endereço para correspondência:

CARLOS AUGUSTO REAL MARTINEZ

Rua Rui Barbosa, 255 - apto 32.

Santo André, São Paulo, CEP: 09190-370.

E-mail: caomartinez@uol.com.br 